\title{
BUSY HOUR TRAFFIC CONGESTION ANALYSIS IN MOBILE MACROCELLS
}

\author{
A. Ozovehe ${ }^{1,}{ }^{*}$, O. U. Okereke ${ }^{2}$, E. C Anene ${ }^{3}$ and A. U. Usman ${ }^{4}$ \\ 1 Trifield Technology Limited, 14 Ishaq MaAjI CRescent, GWagWalada Abuja, NIGERIA \\ 2,3DEPT. of EleCtrical \& ElECTRONICS ENGR., ABUBAKAR TAFAWA BALEWA UNIV. BAUCHI, BAUCHI STATE, NIGERIA

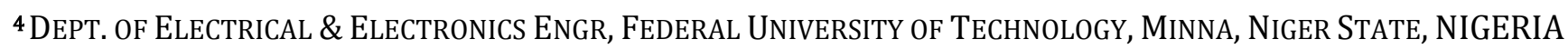 \\ E-mail addresses:1aliyu123oz@gmail.com,2uokereke@gmail.com,3 ejikeanene@yahoo.com, \\ 4 abrahamusman@gmail.com
}

\begin{abstract}
Traffic congestion during busy hour (BH) deteriorates the overall performance of cellular network and may become unmanageable unless effective and efficient methods of congestion control are developed through real live traffic data measurement and analysis. In this work, real live traffic data from integrated GSM/GPRS network was used for traffic congestion analysis. The analysis was carried out on 10 congesting cells using network management system (NMS) statistics data span for three years period. Correlation test showed that traffic channel (TCH) congestion depend only on call setup success rate (CSSR) and BH traffic at cell level. An average correlation coefficient value of 0.9 was observed between TCH congestion and CSSR while 0.6 was observed between TCH congestion and BH traffic. The correlation test is important when selecting input for congestion prediction modeling.
\end{abstract}

Keywords: traffic congestion; correlation coefficient; macrocells and key performance indicator

\section{INTRODUCTION}

All over the world, cellular network operators are faced with the challenges of improving the quality of service (QoS) while increasing capacity and rolling out new services. This has resulted in many congested networks and consequently degradation of QoS due to inadequate provision of the needed resources or underutilization of the available resources.

To cope with subscriber demands and meet Regulator standards, cellular network providers dimension network elements on continuous bases using network management system (NMS) statistics, drive test trailing and customer feedbacks.

However, Nigerian Communication Commission (NCC) quarterly audit reports of GSM network performance had consistently shown that the operators have not been able to meet the set standards [1] due to network congestion [2]. If there is no hardware fault and interference problems, network congestion usually occurs due to insufficient number of radio channels in access network elements [3].

This work used busy hour traffic data of access network from a live network to analyse traffic congestion in some macrocells of GSM/GPRS network. The busy hour of a network is the time when the network processes the highest traffic in a day and it is used to measure network performance, determine the robustness of a network and its dimension [4]. To measure the network performance, the pattern of busy hour traffic is considered for congestion evaluation [5] using key performance indicators (KPIs).

These KPIs are defined for different interfaces and network elements of GSM/GPRS. To cater for subscriber demand, radio frequency (RF) optimization teams use the KPIs to generate quality of service (QoS) reports to ensure minimal congestion over all the interfaces and network elements in order to avoid QoS degradation by maintaining the KPIs under pre-defined threshold [6]. Network congestion leads to poor QoS which affect grade of service (GoS) of the network, particularly during the busy hour of the day [7]. In a loss system, the GoS describes that proportion of calls that are lost due to congestion in the busy hour and can be measured using Eq. (1):

$$
\text { GoS }=\frac{\text { Number_of_lost_calls }}{\text { Number_of_offered_calls }}
$$

Traffic analysis is important for evaluating the performance of an existing networks and network optimization. The events that occur in base transceiver station (BTS) trigger different counters in the base station controller (BSC) and mobile switch center (MSC) memory that are used for performance 
monitoring and network evaluation. Various KPIs that are used to measure network performances are derived with the help of these counters using different formulations [7]. In practice, the performance can be monitored at different sections of the network [8] and in this work the network performance is evaluated at cell levels in terms of resource allocation and resource utilization.

Some of the early works on GSM network elements performance were done mostly on access part of the network particularly at BTS level. For example, [9] proposed a traffic model for mobile network using Markov chain to determine call blocking and handoff failure probabilities when no queuing of new or handover calls is performed while [10] modeled the effect of user mobility on the performance of mobile networks using office hours traffic data. Location updates were analyzed to evaluate the probability of call dropping when handover is needed. Likewise, [11] analyzed seventy eight traffic channels and showed that a single dedicated channel is enough for good QoS. [12] investigated GSM/GPRS system performance using dedicated number of GPRS channels and some idle periods between voice calls for GPRS data packet transfers. Reservation of more channels brings handover failure and dropped call probability to very small values but lack of ordinary channels produces a larger new calls blocking probability.

The work of [13] presented the results of study of DCS1800 Um-interface using daily traffic measurement data. The performance indicators used are Traffic, call setup success rate (CSSR), handover success rate (HOSR), standalone dedicated control channel (SDCCH) and traffic channel (TCH) congestion. The analysis showed the limitations of the system to accommodate extreme offered traffic without adding more resources. A model combining simulations for paging, signaling and traffic channels was developed by [14] to investigate the optimal dimensioning of a finite physical resource allocated across multiple logical channels with multiple traffic types.

Similarly [15] evaluated the performance of GSM1900 Um-interface of two different vendors using daily measurement data for one week. The performance indicators used are peak hour traffic; CSSR; Handover Failure; congestion on control channels (SDCCH blocking rate); congestion on traffic channels (TCH blocking Rate); drop on traffic channels; drop on control channels; cells with TCH congestion rate exceeding $2 \%$ and TCH Mean Holding Time.

In another work, [3] analyzed traffic data from a trunked radio network operated by Ecomm in UK using OPNET model. The findings indicated that traditional Erlang models for voice traffic may not be suitable for evaluating the performance of trunked radio networks. In a related work, [16] formulated a dynamic channel allocation model using Markov chain technique as an improvement on [17].

There is one problem common to all these works at BTS level, exclusive handover channels were employed for easy compliance of QoS standards for ongoing calls and handover failure reduction. However, the disadvantage is that new calls blocking increase as a result of the reduction of available ordinary channels. The solution should have been that resources should be added to maintain GoS of the network as put forward by [18] and supported by the work of [13].

\section{OBJECTIVE OF THE STUDY}

While a whole range of services of GSM technology are in use in Nigeria, it is obvious that the network performance in terms of QoS are degrading which proved that GSM network is either over utilized or under dimensioned. Hence the need for this analysis to identify the cells that are responsible for congestion during busy hour by statistically analyzing traffic data of a live network in order to establish the presence or absence of congestion

\section{METHODOLOGY}

The setup for data collection in GSM/GPRS network comprises of base station subsystem (BSS) and network subsystem (NSS) connected to standalone system called network management system (NMS) as shown in Figure 1.

NMS is the functional entity from which the service provider monitors and controls the entire network. The data used in this work was extracted from the NMS with the help of Ericsson Business intelligent (BI) tools installed on the standalone computer and exported to Microsoft Excel environment.

Air interface (Um) is between the mobile station (MS) and the BTS. Um is defined in the GSM 04.xx and 05.xx series of specifications for signaling, measurement reporting, handover, power control, authentication, authorization, location update and so on. The base station subsystem (BSS) is the section of a traditional cellular telephone network which is responsible for handling traffic and signaling between a mobile phone and the network switching subsystem.

The base transceiver station (BTS) contains the equipment for transmitting and receiving radio signals (transceivers), antennas, and equipment for encrypting and decrypting communications with the BSC.

Abis is the interface between the BTS and BSC. Generally carried by a E1 time division multiple (TDM) circuit. It uses TDM subchannels for traffic (TCH). 


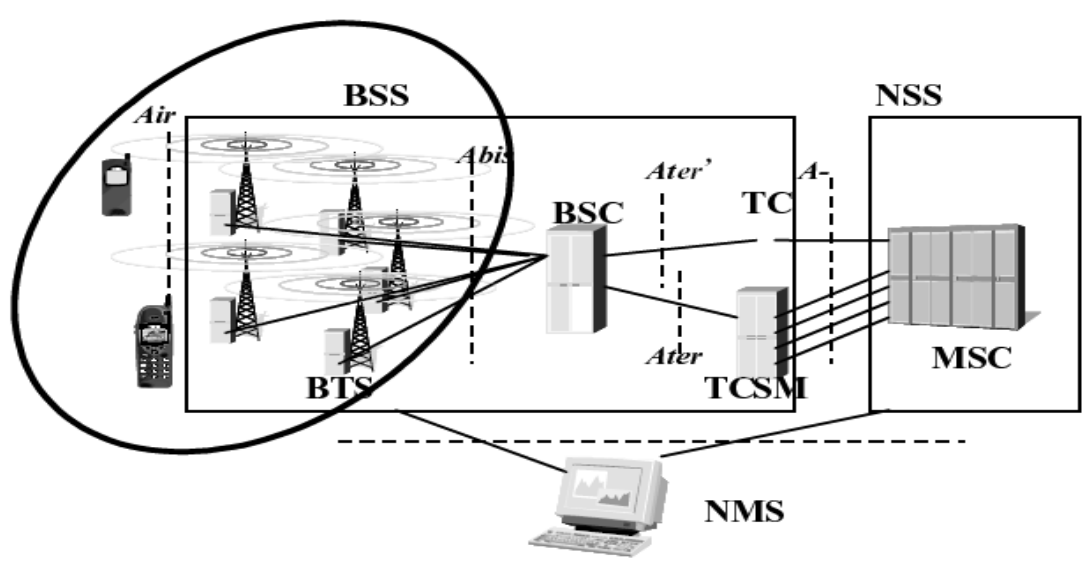

Figure 1: System for collecting traffic data

The Base Station Controller (BSC) manages all the radio-related functions of a GSM network. It is a high capacity switch that provides functions such as MS handover, radio channel.

Ater is the interface between the BSC and transcoder. It is a proprietary interface whose name depends on the vendor.

Transcoder/Sub-Multiplexer (TCSM) is responsible for compression of $64 \mathrm{KBPS}$ from MSC to 13KPS in order to interface with BSC.

A is the interface between the BSC and MSC. It is used for carrying traffic channels and the signaling number 7 (SS7).

Network switching subsystem (NSS) (or GSM core network) is the component of a GSM system that carries out call switching and mobility management functions for phones roaming on the network of base stations.

Mobile Services Switching Centre (MSC) performs the telephony switching functions of the system. It controls calls to and from other telephone and data systems. It also performs such functions as: toll ticketing, network interfacing, common channel signaling, and others.

NMS data used in this work composed of 742 cells, 262 BTSs. Measurements were taken from November, 2012 to September, 2014. Correlation test was used to choose only KPIs that have significant effect on TCH congestion during busy hour.

Correlation coefficient is defined as a number or function indicating the degree of correlation between two variables like $\mathrm{X}$ and $\mathrm{Y}$. In this work, the variables are busy hour traffic and CSSR, HOSR, drop call rate (DCR), SDCCH and TCH Congestions as KPIs to measure the network performance. Eq. (2) defines correlation coefficient as:

$$
\operatorname{Correl}(X, Y)=\frac{\sum(x-\bar{x})(y-\bar{y})}{\sqrt{\sum(x-\bar{x})^{2} \sum(y-\bar{y})^{2}}}
$$

Microsoft excel statistical tools was used for the correlation analysis.

\section{RESULT AND DISCUSSION}

\subsection{Daily Busy Hour Traffic Analysis}

The busy hour traffic data showed that 154 cells experienced congestion out of 742 cells during the period under investigation. In the cell KPIs analysis, 10 worst congesting cells were chosen for busy hour traffic channel (BH TCH) congestion analysis [19] as shown in Table 1.

It can be seen from Table 1 that FC0396B, FC0301C and FC0362C congested continuously for 13 months (January 2013 - February 2014), FC0393C, FC0430C and FC0494B congested continuously for 9 months (April 2013 - February 2014) without intervention by the network provider while the rest of the cells congestion were resolved and get congested again for the period of 23 months investigated in this study. Hence, there is need to know to investigate these cells to know if they congested as a result of user demand or fault. Table 2 shows the detailed configuration of the 10 congested cells. The total defined transceivers (TRX), TCH availability (\%) and traffic (Erlang) is 2, 100 and 14 respectively for the cells. It is obvious from Table 2 that these cells are designed to carry maximum traffic of 14 Erlang except cellFC0496B, FC0430C and FC0038B which are designed to carry 26TCH at $2 \%$.However, these cells carried more traffic during busy hour than what they are designed for, hence the cells congested due to demand.

\subsection{Tch Congestion Correlation Test}

Using the BTS KPIs and BH traffic, the correlation test showed that the ten worst cells behaved differently in terms of KPIs that have strong correlation with TCH congestion during busy hour which implied that they must be investigated differently based on their correlation test results. 
Busy Hour Traffic Congestion Analysis in Mobile Macrocells, A. Ozovehe, 0. U. Okereke, E. C Anene \& A. U. Usman

Table 1: Ten Worst Congesting Cells

\begin{tabular}{ccccccccccc}
\hline \multirow{2}{*}{ Dates } & \multicolumn{10}{c}{ Cell Ids } \\
\cline { 2 - 11 } & FC0396B & FC0301C & FC0362C & FC0385B & FC0393C & FC0430C & FC0494B & FC0496B & FC0038B & FC0725C \\
\hline Jan-13 & 3.98 & 60.18 & 80.01 & 2.14 & 4.35 & 78.95 & 46.04 & 69.59 & 3.7 & - \\
Feb-13 & 14.44 & 46.81 & 23.86 & 27.91 & 13.83 & 24.64 & 19.92 & - & 14.67 & - \\
Mar-13 & 50.87 & 3.96 & 62.71 & - & - & - & - & 20.4 & 2.41 & 44.16 \\
Apr-13 & 20.78 & 30.47 & 50.41 & 32.12 & 19.74 & 24.28 & 55.9 & - & 7.53 & - \\
Jun-13 & 15.31 & 48.24 & 59.02 & 9.39 & 21.63 & 28.87 & 33.77 & 10.23 & 2.39 & - \\
Jul-13 & 10.02 & 53.61 & 69.77 & 4.11 & 14.72 & 13.31 & 17.12 & 7.65 & - & 30.85 \\
Aug-13 & 7.15 & 37.83 & 67.51 & 2.17 & 18.28 & 18.13 & 16.78 & 3.19 & - & 38.36 \\
Sep-13 & 10.18 & 33.95 & 65.27 & - & 19.57 & 5.26 & 14.01 & 1.86 & - & 16.22 \\
Oct-13 & 9.99 & 52.04 & 61.52 & 3.1 & 21.07 & 12.43 & 21.69 & 6.05 & 16.97 & 2.76 \\
Nov-13 & 13.8 & 58.28 & 63.61 & 3.03 & 11.34 & 3.68 & 36.6 & 4.32 & - & - \\
Dec-13 & 3.42 & 74.94 & 84.54 & 13.31 & 18.45 & 9.28 & 31.63 & 32.65 & - & - \\
Feb-14 & 16.65 & 71.21 & 80.59 & 14.57 & 9.69 & 82.61 & 54.85 & 83.13 & 17.22 & 19.15 \\
\hline
\end{tabular}

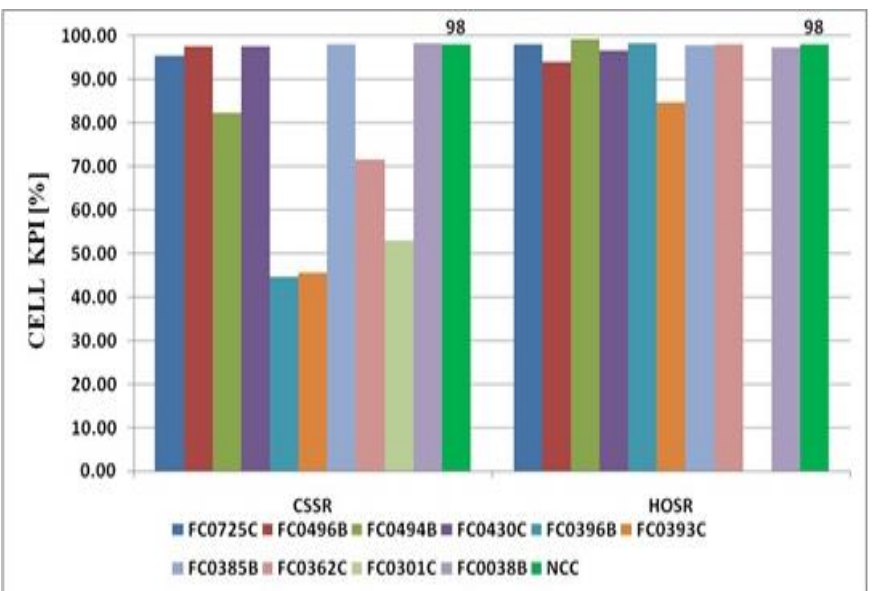

Figure 2: CSSR and HOSR for ten worst Cells during Max. Traffic

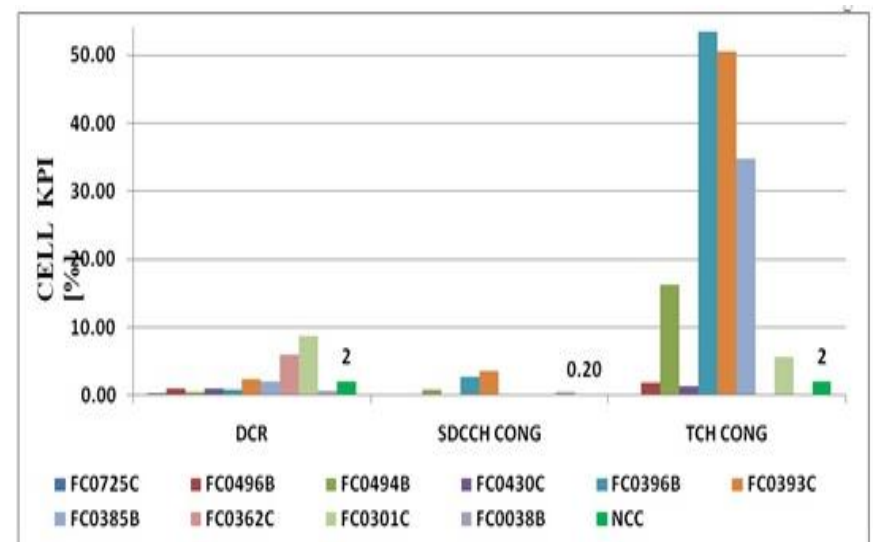

Figure 3: DCR, SDCCH CONG and TCH CONG for ten worst Cells during Max. Traffic

However, we selected 4 cells that passed correlation test coefficient factor of 0.5 and above to know which KPI has strong correlation with TCH congestion at cell level. The result of the test for the four cells is summarized in Table 3 . Table 3 shows that $\mathrm{BH}$ TCH congestion has strong correlation with CSSR and busy hour traffic for the four cells. Hence, the knowledge of CSSR and busy hour traffic can be used to predict TCH congestion using artificial intelligent techniques. Given the result of the correlation test, the maximum daily $\mathrm{BH}$ traffic carried by the ten cells and with its impact on CSSR and TCH congestion is shown in Figure 2 and Figure 3. From Figure 2 and 3, most of the cells CSSR and TCH Congestion degraded when they carried maximum traffic while Cell 396B, 393C and 301C suffered worst KPIs degradation. The behaviour of the ten worst cells when they experienced worst TCH CONG and the effect on other KPIs during the period is shown in Figure 4 to 6.

All the cells CSSR degraded and their ability to carry traffic is limited when they experienced worst TCH CONG while Cell 496B, 430C, 396B and 038B recorded worst KPIs degradation. This shows that traffic channel of these cells are not properly dimensioned and hence the need to add physical radio resources to the cells in order to resolve the congestion.

Table 2: Daily BH TRAFFIC (Erlang) for the ten Worst Cells, Total defined $(T R X)=2$, TCh avilability $=100 \%$, Defined Traffic $=14$.

\begin{tabular}{ccc}
\hline Cell & Max Carried & Average Carried Traffic \\
\hline FC0725C & 25.6 & 15.01 \\
FC0496B & 43.93 & 18.11 \\
FC0494B & 21.68 & 13.61 \\
FC0430C & 64.03 & 37.9 \\
FC0396B & 21.59 & 12.75 \\
FC0393C & 19.82 & 13.85 \\
FC0385B & 47.65 & 13.08 \\
FC0362C & 27.66 & 15.77 \\
FC0301C & 34.99 & 17.12 \\
FC0038B & 54.41 & 21.72 \\
\hline
\end{tabular}




\section{Busy Hour Traffic Congestion Analysis in Mobile Macrocells, A. Ozovehe, 0. U. Okereke, E. C Anene \& A. U. Usman}

Table 3: Summary of TCH Congestion Correlation Test TCH Congestion Correlation

\begin{tabular}{c|c|c|c|c|c}
\hline Cell & CSSR & DCR & HOSR & SDCCH CONG & Traffic \\
\hline FC0725C & 0.9 & 0.2 & 0.1 & 0.4 & 0.5 \\
FC0393C & 1.0 & 0.1 & 0.4 & 0.1 & 0.6 \\
FC0362C & 0.9 & 0.7 & 0.1 & 0.0 & 0.7 \\
FC0301C & 0.7 & 0.6 & 0.1 & 0.4 & 0.6 \\
\hline Average & 0.9 & 0.4 & 0.2 & 0.2 & 0.6 \\
\hline
\end{tabular}

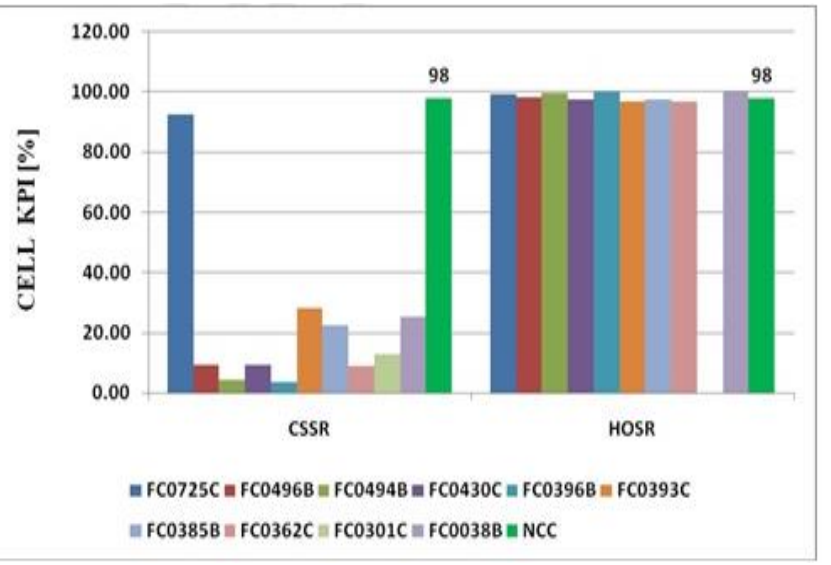

Figure 4: CSSR and HOSR for the Cells during Worst TCH CONG

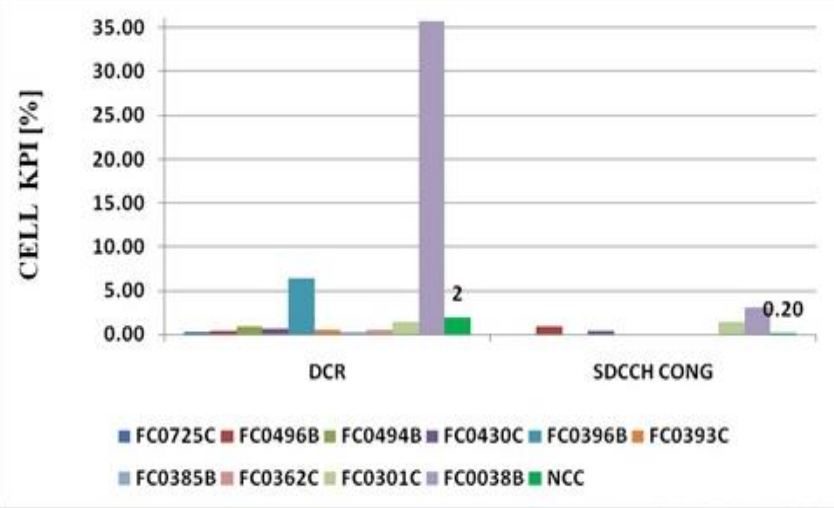

Figure 5: DCR and SDCCH CONG for the Cells during Worst TCH

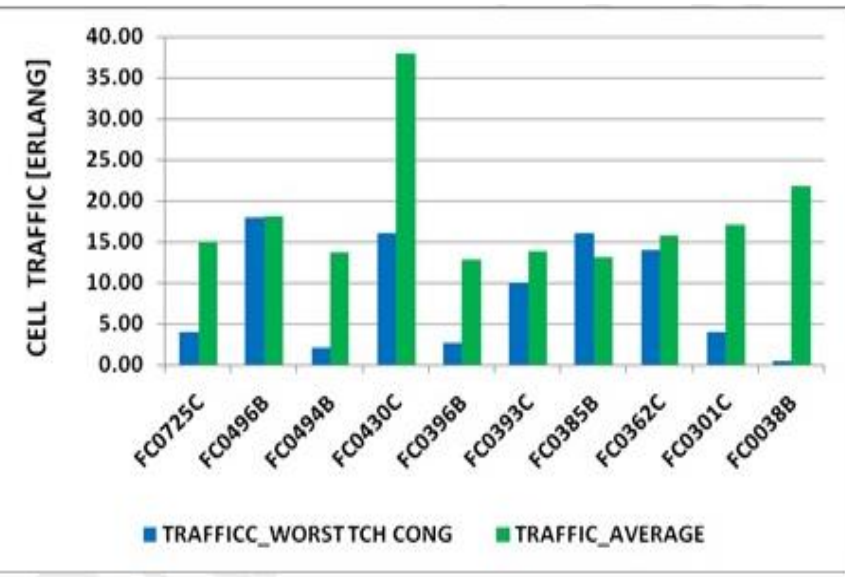

Figure 6: Traffic Average and Traffic Carried by the Cell worst TCH CONG

\section{CONCLUSION}

The analysis of traffic channels in an existing network showed that TCH congestion beyond the acceptable $2 \%$

Nigerian Journal of Technology, threshold for traffic channel (TCH) occurred in 154 cells out of 742 cells investigated.

The analysis of the ten most congested showed that slow response to congested cells is the major setback that affects the QoS as some cells congested continuously over one year.

The busy hour TCH congestion correlation analysis showed that the congestion depends on CSSR and BH traffic. The CSSR and BH traffic has an average correlation coefficient of 0.9 and 0.6 respectively for the cells. The strong correlation showed that the knowledge of CSSR and busy traffic can be used to predict TCH congestion which is crucial for cellular network optimization and resource management.

\section{REFERENCES}

[1] Nigerian Communications commission Website, Retrieved October 25, 2015, from: HTTP://WWW.NCC.GOV.NG/INDEX.PHP?OPTION=COM_CO NTENT\&VIEW $=$ ARTICLE\&ID $=332 \&$ ITEMID $=104$

[2] O. Aliyu, Okereke O. U. and Anene E.C, Literature Survey of Traffic Analysis and Congestion Modeling In Mobile Network, IOSR Journal of Electronics and Communication Engineering (IOSR-JECE) e-ISSN: 2278-2834,p- ISSN: 22788735.Volume 10, Issue 6, Ver. I (Nov - Dec .2015), PP 31-35 www.iosrjournals.org 2015.

[3] V. Božidar, Modeling and Characterization of Traffic in a Public Safety Wireless Network, Master of Applied Science Thesis, Simon Fraser University, fall 2006.

[4] E. O. Oladeji, E. N. Onwuka and M. A. Aibinu, Determination of Voice Traffic Busy Hour and Traffic Forecasting in Global System of Mobile Communication (GSM) in Nigeria, 2013 IEEE 11th Malaysia International Conference on Communications 26th - 28th November, Kuala Lumpur, Malaysia, 2013.

[5] S. Kyriazakos, G. Karetsos, E. Gkroustiotis, C. Kechagias and P. Fournogerakis, Congestion Study and Resource Management in Cellular Networks of present and Future Generation, IST Mobile Summit 2001, Barcelona, Spain, 9-12 September 2001.

[6] Z. Jens, Radio Resource Management for Wireless Networks, Artech House Inc., 2001.

[7] B. Haider, M. Zafrullah, and M. K. Islam, Radio Frequency Optimization and QoS Evaluation in Operational GSM Network, Proceedings of the World Congress on Engineering and Computer Science, Vol. I WCECS 2009, October 20-22, 2009, San Francisco, USA, 2009.

[8] I. Kennedy, Lost Call Theory, Lecture Notes, ELEN5007 - Teletraffic Engineering, School of 


\section{Busy Hour Traffic Congestion Analysis in Mobile Macrocells, A. Ozovehe, 0. U. Okereke, E. C Anene \& A. U. Usman}

Electrical and Information Engineering, University of the Witwatersrand, 2005.

[9] B. Jabari, Teletraffic Aspects of Evolving and NextGeneration Wireless Communication Networks, IEEE Personal Communications Mag., Vol. 3, No.6, Dec., pp4-9, 1996.

[10] Y. C. Min, S. K. Tai, S. C. Ho and Dan, K. S. Modeling of Terminal Mobility to Evaluate the Number of Location Updates, 1997 IEEE International Conference on Volume 3, Issue , 8-12 Jun 1997 Page(s):1266 - 1270.

[11] A. M. Francisco and Luis, M. C., Mobility Effects on Teletraffic in GSM, Instituto de Telecomunicações / Instituto Superior Técnico, Technical University of Lisbon, 1999.

[12] A. Hugo, C. José and M. C. Luis, Analysis of a Traffic Model for GSM/GPRS, Instituto de Telecomunicações/Instituto Superior Técnico, Technical University of Lisbon, 2000.

[13] K. N. Papaoulakis, D. Nikitopoulos, E. Gkroustiotis, C. Kechagias, C. Karambalis and G. Karetsos, A Comprehensive Study and Performance Evaluation of Operational GSM and GPRS Systems under Varying Traffic Conditions, Telecommunications Laboratory National
Technical University of Athens Heroon Polytechniou 9, 15773 Athens, Greece.

[14] K. Allen, P. Fitzpatrick and M. Ivanovich, Joint Traffic Signaling Capacity Analysis in GSM, Telstra Research Laboratories, Clayton, Vic 3168

[15] M. Boulmalf and S. Akhtar, Performance Evaluation of Operational GSM's Air-Interface (Um), in Proc of Applied Telecommunication Symposium," Orlando, Florida, pages 62-65, March 2003.

[16] O.A. Ojesanmi, T. O. Oyebisi, E. O. Oyebode, and O. E. Makinde, Performance Analysis of Congestion Control Scheme for Mobile Communication Network, International Journal of Computer Science and Telecommunications, Volume 2, Issue 8, November 2011.

[17] J. Xiaolong and M. Geyong, Modelling Priority Queueing Systems with Multi-Class Self-Similar Network Traffic, Proceedings of ICC'07, pp. 13-19

[18] Kennedy I. Why Traffic Measurement, School of Electrical and Information Engineering, University of the Witwatersrand, 2003.

[19] Ericsson, Root Cause Analysis for Key Performance Indicators (KPI) for GSM, Networks, REP00271 A, ESA/SK 06:0027. 